\title{
Correction to: After Deployment, Adaptive Parenting Tools: 1-Year Outcomes of an Evidence-Based Parenting Program for Military Families Following Deployment
}

\author{
Abigail H. Gewirtz ${ }^{1}$ - David S. DeGarmo ${ }^{2}$. Osnat Zamir ${ }^{3}$
}

Published online: 2 November 2017

(C) Society for Prevention Research 2017

Correction to: Prevention Science (2017)

https://doi.org/10.1007/s11121-017-0839-4

The authors would like to indicate the below corrections to Table 2 of the above referenced article.

The note is missing, and the CACE indirect coefficients should be $.18^{* *}$ and $.16^{* *}$ instead of $.16^{* *}$ and $.15^{* *}$. The corrected table is shown in this article.

The online version of the original article can be found at https://oi.org/ 10.1007/s11121-017-0839-4

\footnotetext{
Abigail H. Gewirtz

agewirtz@umn.edu

1 University of Minnesota, Minneapolis, MN, USA

2 University of Oregon, Eugene, OR, USA

3 Hebrew University of Jerusalem, Jerusalem, Israel
} 
Table 2 Standardized indirect effects and confidence intervals for ITT and CACE

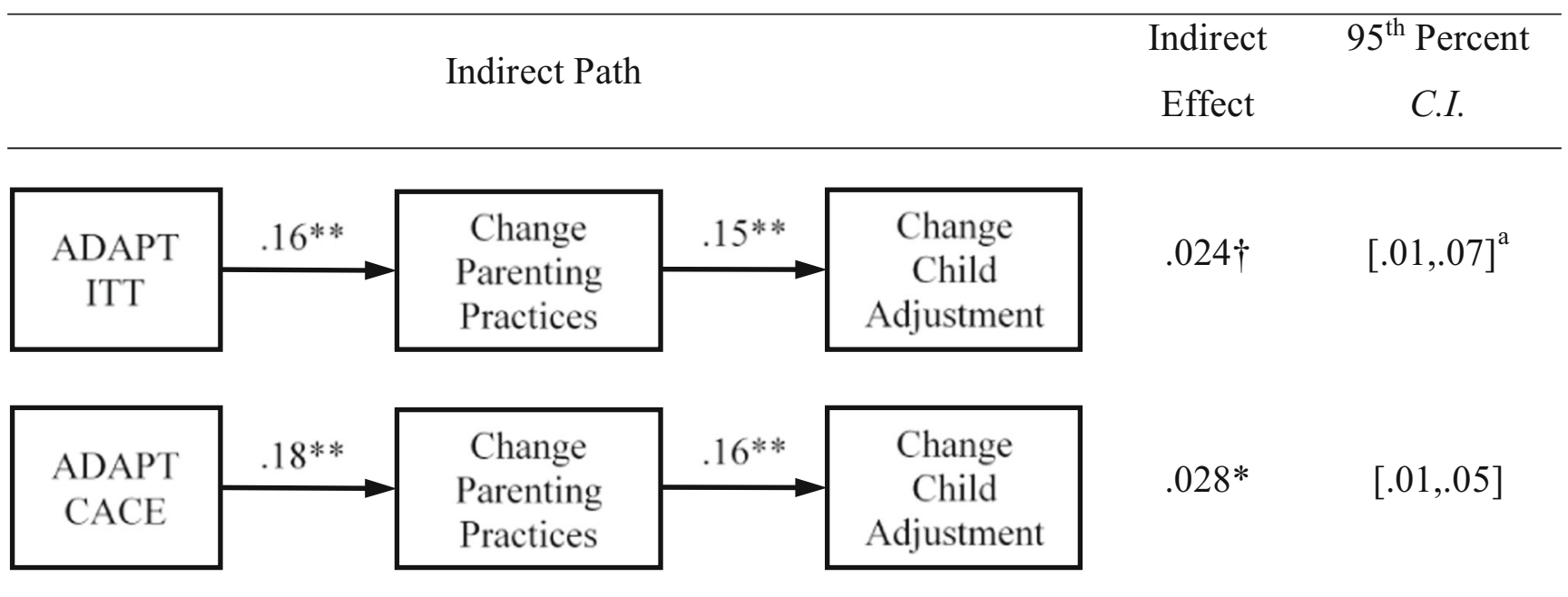

${ }^{a}$ Bias corrected bootstrapped confidence intervals with 1000 draws

$* * * p<.001, * * p<.01, * p<.05, \dagger p<.10$ 